\title{
La gestión del conocimiento en el marco de la prestación de servicios de atención prenatal dirigida a adolescentes primigestas
}

\author{
The management of knowledge in the context of pre-natal \\ care directed to primigest teens
}

\author{
Hilda E. Valencia-Marroquín* \\ Programa Universitario de Investigación Interdisciplinaria en Salud, Dirección General de Investigación, \\ Universidad de San Carlos de Guatemala.
}

*Autor al que se dirige la correspondencia: hildvalencia@gmail.com

Recibido: 19 de mayo 2015 / Revisión: 06 de julio 2015 / Aceptado: 28 de junio 2017

\section{Resumen}

$\mathrm{E}$ n los últimos años no solo han cambiado los métodos y técnicas para gestionar el conocimiento sino también los criterios de cómo valorarlo. De igual forma, esto ha sucedido con el concepto de salud pública, donde los cambios realizados y las redefiniciones se han dado tanto en sus acciones como en el sujeto de intervención. La gestión del conocimiento en el ámbito privado se ha utilizado con éxito, principalmente en empresas transnacionales, quienes se han enfocado en la generación del conocimiento individual de sus empleados, haciendo énfasis en compartir los elementos que hacen posible la generación de este conocimiento; situación que es vital para el desarrollo del capital humano y la creación de una cultura organizacional que permita alcanzar altos estándares en la producción de bienes y servicios. En el ámbito de la salud pública, la gestión del conocimiento debe ser orientada al perfeccionamiento de procesos de impacto, mediante la articulación de la gestión de la información, la tecnología y los recursos humanos; de esta forma contribuir a la exploración y distribución del conocimiento existente en función de los procesos esclarecidos sobre la base del uso intensivo de las redes y de la tecnología; provocando con ello, la implantación de mejoras continuas, innovación en la práctica, integración funcional, desarrollo de servicios contextualizados, facilitando con ello el incremento de la competitividad y sostenibilidad de los servicios en donde se implante. En este ensayo se desarrolla la propuesta para aplicar la gestión del conocimiento en la atención prenatal de la adolescente primigesta.

\section{Abstract}

Tn recent years not only have changed the methods and techniques to manage knowledge, but also the criteria of Ihow to value it. The same has happened with the concept of public health, where changes made and redefinitions have been as much in their actions as in the subject of intervention. Knowledge management in the private sector has been used successfully, mainly in transnational companies, which have focused on the generation of individual knowledge of their employees, emphasizing in sharing the elements that make possible the generation of this knowledge. This situation is vital for the development of human capital and the creation of an organizational culture that allows to reach high standards in the production of goods and services. In the field of public health, knowledge management should be oriented towards the improvement of impact processes, through the articulation of information, technology and human resources management; thus contributing to the exploration and distribution of existing knowledge in the light of enlightened processes based on the intensive use of networks and technology, creating continuous improvements, innovation in practice, functional integration, development of contextualized services, thereby facilitating the increase of competitiveness and sustainability of the services in which it is implanted. This essay develops the proposal to apply the knowledge management in prenatal care of the pregnant adolescent and especially to the one who is pregnant for the first time. 


\section{Introducción}

El enfoque de la gestión del conocimiento, en su inicio, estuvo dirigido a la creación y mantenimiento de contenedores de información. Posteriormente resaltó la importancia en las personas y la cultura de la institución. En la actualidad, la institución es un aliado de la generación del conocimiento individual de sus empleados y su énfasis está en compartir los elementos que hacen posible la generación del conocimiento (Archanco, 2013).

La importancia de la gestión del conocimiento en la salud pública está relacionada con las capacidades de los individuos de actuar y resolver problemas con la información disponible y con la creación de flujos de información y comunicación altamente flexibles y cambiantes (Urra, 2008).

Lo anterior, exige una respuesta de los actores involucrados y un ciclo de creación de conocimientos para la acción, en forma mucho más rápida a partir de nuevos conocimientos creados (Urra, 2008).

Es decir, se trata de un balance entre la eficiencia y la eficacia para resolver sus problemas usando la información y el conocimiento. Por consiguiente, la gestión del conocimiento en una institución busca perfeccionar en forma continua la infraestructura técnica, logística y organizacional; garantiza su uso eficiente y seguro; fortalece la interacción de una red intra e interinstitucional; logra la sostenibilidad del sistema mediante actualización de recursos y su uso racional; controla y, evalúa en forma sistemática los avances de acuerdo con metas establecidas como elemento natural de los procesos (Urra, 2008).

Otros aspectos importantes de mencionar son los siguientes: la gestión del conocimiento propicia mecanismos que facilitan el establecimiento de una red de relaciones adecuadas, mediante la cual, se posibilita la activación de la dimensión colectiva del conocimiento y con ello la promoción de su generación y transferencia en forma contextualizada y con pertinencia cultural.

La gestión del conocimiento produce la interacción de los individuos lo que permite la articulación del conocimiento explícito y tácito, promoviendo la creación de un sentido de comunidad entre los usuarios, usuarias y proveedores, ayudándoles a mantenerse actualizados en su campo de conocimiento.

Una institución de salud debe tener la capacidad de generar nuevos conocimientos y de diseminarlos entre sus miembros para concretarlos en productos, servicios y sistemas que permitan dar respuesta a los problemas de salud prevalentes.

\section{Contenido}

Según Davenport y Prusak (1998), el conocimiento es un conjunto de experiencias, valores, información, percepciones e ideas que crean una estructura mental para evaluar e incorporar nuevas experiencias, ideas e información; siempre y cuando permita comparar consecuencias, conectar y conversar.

El significado que cada sociedad confiere al conocimiento es producto de construcciones sociales, que articuladas con sus contenidos axiológicos generan esquemas de decisión que son utilizados por los sistemas sociales para administrar el saber. Lo anterior permite valorar, estimular, censurar o reprimir ciertas formas de conocimiento; por ello, en los últimos años, el mundo no solo ha cambiado sus métodos y técnicas para gestionar el conocimiento, sino también sus criterios de cómo valorarlo (Red Colombiana de Investigación en Política y Sistemas de Salud, 2009).

Según Lundvall (1999) en donde cita a Lundvall y Johnson (1994), clasifica el conocimiento en cuatro categorías: saber qué, saber por qué, saber cómo y saber quién, situación importante de especificar en un proceso de transferencia del conocimiento.

El saber qué se refiere a los hechos; el saber por qué es el conocimiento importante para el desarrollo tecnológico. El saber cómo, se refiere a la habilidad de hacer algo, se relaciona con la producción y otras actividades del ámbito económico. El saber quién implica información acerca de quién sabe qué y quién sabe cómo hacer qué (Lundvall, 1999).

Cabe considerar que el dominio de estas clases de conocimiento se realiza por medio de diversos canales para su externalización.

Ahora bien, el conocimiento presenta un ciclo de vida que se relaciona con el intercambio generado entre los individuos de la organización, estructurándose en un conjunto de operaciones interdependientes: creación, captura, procesamiento, diseminación, adquisición y aplicación; por consiguiente, el ciclo se cierra e inicia al retroalimentar el sistema.

El ciclo de vida del conocimiento se integra mediante las siguientes operaciones (Bürkli, 2011):

Creación. Proceso en el que se genera nuevo conocimiento en la mente de los miembros de la or- 
ganización. (El conocimiento involucrado puede ser explícito o tácito).

Captura. Difiere según el tipo de conocimiento. Para el conocimiento explícito, se hace mediante su codificación o representación en forma de documentos. Para el conocimiento tácito explicable, se lleva a cabo por medio de la verbalización y luego se codifica en forma de documentos.

Si el conocimiento es tácito, es decir no explicable, su captura se realiza a través de estrategias de socialización entre el sujeto que lo posee y el resto de la comunidad.

Procesamiento. Valora la pertinencia de la información para la consecución de los objetivos de la organización. Se someten documentos al análisis documental de forma y contenido y posteriormente se incorpora a un sistema documental para facilitar el acceso a los miembros de la organización.

Diseminación. Se realiza para que el conocimiento de la organización llegue a los miembros de la comunidad de acuerdo con sus necesidades.

Adquisición. Se centra en la forma como los individuos asimilan y comprenden el conocimiento de la organización, puede ser resultado de la lectura de documentos o por socialización.

Aplicación. Se produce cuando el conocimiento adquirido es aplicado y reutilizado en la práctica diaria por los miembros de la organización. La reutilización puede ser en contextos diferentes al que se originó el conocimiento en este proceso se realiza una reinterpretación que facilita la creación de nuevo conocimiento.

Es evidente entonces que el conocimiento operativo en una organización se genera a través de una estrategia organizacional que comprende tanto los procesos, como la competencia de sus trabajadores, normativas y tecnología existente (Bürkli, 2011).

Lo anterior significa que el conocimiento asociado a una persona y a una serie de habilidades personales se convierte en sabiduría y el conocimiento asociado a una organización y a capacidades organizativas se convierte en capital intelectual (Bürkli, 2011).

En la expresión sociedad del conocimiento popularizada por Sakaiya en 1995, se describe una visión de la estructura de la sociedad venidera, en una anticipada historia del futuro (Boisier, 2002). En ella incorpora como uno de los principales componentes de las relaciones de producción, al conocimiento organizacional; inclusive más allá de la Tierra (Recursos Naturales), el Capital (Recursos Financieros) y el Trabajo (Recursos Humanos), que han sido tradicionales variables de la función de producción (Argote \& Ingram, 2000).

Según Sakaiya (1991), la sociedad del conocimiento nace como consecuencia de cambios que aparecen en el escenario social y empresarial en donde una ética y una estética diferentes conducen a una sociedad con nuevos paradigmas.

En tal sentido, Grant (1996), explica que en el nuevo siglo se introdujo de lleno la cultura del conocimiento, debido a que el patrimonio intelectual adquiere cada día más valor, entendido este como el conjunto de informaciones que reportan ventajas competitivas sobre los demás.

Según Sveiby (2001), el principal valor de las empresas ya no reside en su capital tangible, sino en lo que se coincide en llamar capital intelectual o capital intangible. Por lo tanto, este nuevo valor le proporciona a la empresa una clara ventaja competitiva sobre las demás y justifica la diferencia, entre su valor en el mercado y su valor contable; es decir, que la cualificación de los empleados de una empresa (como capital intangible) estará directamente relacionada con el valor de mercado de la misma.

Por lo tanto, los elementos a considerar en la gestión del conocimiento deben ser: el capital intelectual, la cultura organizacional y la tecnología de la información.

Como capital intelectual, se entiende el valor tangible de la organización. Sus elementos básicos son los objetivos, recursos y el entorno organizacional; siendo el liderazgo, la herramienta fundamental de enlace entre los objetivos y los recursos (Bürkli, 2011).

La cultura organizacional es caracterizada por un patrón complejo de creencias, expectativas, ideas, valores, actitudes y conductas compartidas por los integrantes de una organización y el personal los tiene incorporados en sus mentes y corazones. Esta cultura debe reflejar la visión, estrategias y experiencias de los empleados al ponerlas en práctica.

Con relación a clima laboral es importante diferenciarlo de cultura, porque el primero está enfocado a la satisfacción del empleado con el trato de la empresa y mide el nivel de confianza, respeto, justicia y orgullo, así como las relaciones con los líderes de la organización. 
La tecnología de la información y comunicación, es el conjunto de avances tecnológicos que proporciona la informática, las telecomunicaciones y que comprenden los desarrollos relacionados con los ordenadores, internet, telefonía, entre otras (Bürkli, 2011).

De modo que la tecnología se refiere a la aplicación de los conocimientos científicos para facilitar la realización de las actividades humanas. La información abarca los datos a los cuales se les ha dado un sentido y es útil para los humanos y la comunicación es la transmisión de mensajes y la realimentación de los contenidos.

Ahora bien, el capital total de una empresa lo compone el capital tangible y el capital intangible (intelectual) (Bürkli, 2011).

Con relación al capital intelectual, Ordoñez (1999), refiere que se compone de múltiples factores que giran en torno a un nuevo concepto, el de la información, y el del conocimiento. Se trata de activos poco cuantificables, como la formación técnica o especializada de los empleados, su experiencia, los índices de fidelidad de sus clientes, la propiedad intelectual, las patentes, las nuevas formas de hacer negocios o las técnicas para captar nuevos clientes, o de proveer servicios.

Es por eso que la organización para ser competitiva de forma sostenida en el tiempo, deberá identificar, crear, almacenar, transmitir y utilizar de forma eficiente el conocimiento individual y colectivo de sus trabajadores (Serradell \& Juan, 2003) y de esta forma poder resolver problemas, mejorar procesos o servicios y sobre todo, aprovechar nuevas oportunidad de negocio.

Ese mecanismo del conocimiento dentro de las organizaciones fue descrito por Davenport y Prusak (1998), como un proceso dividido en tres etapas: codificación del conocimiento, transferencia del conocimiento y generación del conocimiento.

Resulta claro por ende, que se necesita el establecimiento de procesos mediante los cuales se obtengan, desplieguen o utilicen una variedad de recursos básicos para apoyar los objetivos de la organización, que Koontz y Weihrich (1998), le denominaron gestión.

En la práctica, al referirse a los términos gestión de la información y gestión del conocimiento se hace complejo trazar una línea divisoria entre ellos; porque la gestión de información se orienta a obtener información correcta, en forma adecuada para la persona indicada, al costo adecuado, en el momento oportuno, en el lugar indicado para tomar la acción precisa (Woodman, 1985).

La gestión del conocimiento es el proceso sistémico y específico de una organización, cuya finalidad es adquirir, organizar y comunicar, tanto el conocimiento tácito como el explícito de los empleados, en donde su objetivo primordial es que éste sea utilizado por otros empleados y de esta forma ser más productivos y eficaces en su trabajo (Gil-Montelongo, López-Orozco, Molina-García, \& Bolio-Yris, 2011).

Es decir, que el objetivo básico de la gestión del conocimiento es la creación del valor, independientemente del capital intelectual, de difícil cuantificación en los balances y su propósito es mejorar los resultados de la empresa en el corto, mediano y largo plazo para la obtención de mayores beneficios.

De este modo, el valor que genera permite una eficaz circulación de las ideas o las informaciones y, muy especialmente, del llamado conocimiento tácito, que es la verdadera fuente de competitividad y sostenibilidad de las empresas y de progreso en las sociedades avanzadas.

Por ello, el desarrollo de sus procesos debe permitir determinar las necesidades de conocimiento en la actualidad y en el futuro de la organización; identificar y suplir las carencias; garantizar la aplicación del mismo con eficacia y eficiencia de manera productiva, para contribuir al alcance de las metas y objetivos planteados para la transformación de la realidad objetivo.

Las organizaciones que desarrollan una gestión del conocimiento tienen capacidad de cohesionar y generar sentimiento de identidad, sensibilidad al entorno con el fin de aprender y adaptarse, tolerancia con el pensamiento y la experiencia no convencional, precaución financiera para retener los recursos que aseguran flexibilidad imprescindible en el entorno (Fernández, 2000).

Por consiguiente, la gestión del conocimiento debe considerarse como: “.... un hecho social con base en la experiencia colectiva de sus empleados" y comprende los "procesos estratégicos de producción, difusión, transferencia y popularización”. Siendo importante en la producción de conocimientos la "recopilación de datos, procesamiento, clasificación y documentación" (Davenport \& Prusak, 1998).

La gestión del conocimiento requiere también conocer las capacidades institucionales y la estrategia de la organización donde va a ser desarrollado (Agencia Nacional de Infraestructura, 2012).

Según algunos autores la gestión de la información se supedita a la gestión del conocimiento, pero es criterio de la autora, que ambas se complementan. Una de las tareas prioritarias de la gestión del conocimiento, según Roldán (2000) es la definición de los beneficios que se pueden alcanzar y que suelen variar en función 
de las estrategias de cada organización y que fundamentalmente se basan en los siguientes objetivos: mejorar procesos, generar innovación y desarrollo de nuevos servicios y productos, mejorar relación con los clientes.

Entre las aportaciones que la gestión del conocimiento ha dotado a las empresas se mencionan las siguientes: fomento de investigación y desarrollo, con orientación hacia la innovación; mayor contextualización y caracterización de los mercados y clientes; valoración de las personas y fomento de la cultura organizacional; alineación de procesos y sinergias (Martínez, 2011). Para la selección del modelo y de las actividades para implementar la gestión del conocimiento debe considerarse no solo el ciclo del conocimiento específico de la organización sino también el horizonte de tiempo.

Por lo anterior, el modelo debe de estar ajustado a las metas de largo y corto plaz". Los modelos cíclicos son preferidos más que los lineales y las actividades deben tener sentido dentro del contexto de la gestión, además del establecimiento de metas concretas (Agencia Nacional de Infraestructura, 2012).

De acuerdo con Senge (2011), las organizaciones que aprenden poseen cinco características principales: sistemas de pensamiento, dominio personal, modelos mentales, visión compartida y aprendizaje en equipo.

Gibbons y colaboradores (1997), proponen dos modos en la producción del conocimiento que a continuación se definen:

Modo I. Este modelo distingue entre lo fundamental y lo aplicado; esto implica una distinción operacional entre el núcleo teórico y otras áreas del conocimiento; de tal manera, que las comunidades tratan de que sus teorías se vuelvan marcos de referencia obligados para todos los trabajadores de un campo determinado.

Modo II. El contraste relevante se enfoca en la solución de problemas siguiendo los códigos y la práctica de una disciplina en particular y la solución de problemas que se organizan alrededor de una aplicación particular.

Las características de este modo son: el contexto de aplicación; la transdisciplinariedad, heterogeneidad y flexibilidad. Este modo se caracteriza por ser reflexivo, fundamentado en valores e intereses de los grupos. Su difusión es dirigida a diferentes contextos y su evaluación es en función a controles de calidad.

Existen modelos de gestión del conocimiento que tienen dentro de sus objetivos generar valor en las organizaciones, podemos mencionar: KPMG Consulting (1987), Creación del Conocimiento (1995), Socio técnico (1998), Dinámico de rotación del conocimiento (1999) e Integración de tecnología (2000).

Otros modelos existentes se enfocan en la clasificación y medición del capital intelectual entre ellos se señalan: Balanced Business Scorecard (1992), Navegador de Skandia (1997), Technology Broker (1996), Canadian Imperial Bank (1996), Universidad de West Ontario (1996), Dirección por competencias (1998), Intelect (1998), Nova (2000), entre otros.

Con excepción del modelo propuesto por Nonaka y Takeuchi (1995) la mayoría de modelos coinciden, con mayor o menor dispersión y claridad, en establecer tres fases básicas en la implantación de cualquier sistema de gestión del conocimiento, siendo estas: diagnóstico organizacional, diseño y desarrollo del sistema para la creación y gestión del conocimiento, evaluación y seguimiento de los resultados.

El modelo de conversión de conocimiento se fundamenta en la existencia de una interacción social y un intercambio entre conocimiento tácito y el explícito durante las actividades creativas de las personas. De esta forma, el conocimiento humano se crea y expande. Propone opciones metodológicas para expresar lo inexpresable, diseminar el conocimiento y generar nuevo conocimiento y su análisis se basa en la experiencia japonesa y está relacionada con el enfoque organizacional. Para este modelo, el conocimiento implícito o tácito es el conocimiento que no se ha documentado ni lo ha hecho implícito quien lo usa y controla. El conocimiento codificado es potencialmente conocimiento compartido mientras el no codificado permanece individual hasta que se haya aprendido mediante interacción directa con el poseedor (Nonaka \& Takeuchi, 1995).

El objetivo de la codificación del conocimiento es colocarlo en alguna forma legible, entendible y organizado, para que pueda ser utilizado por todas las personas que necesiten de él (Rodríguez, 2006).

Para Nonaka y Takeuchi (1995), el contexto del trabajo es el que permite la construcción de un escenario de aprendizaje real, en el que los actores involucrados, mediante la aplicación de la teoría en la práctica validan el conocimiento o producen conocimiento nuevo.

En este modelo, el "descubrimiento" del conocimiento es un proceso que permite ampliar en cantidad y calidad un almacén de conocimiento y se lleva a cabo a través de una serie de eventos que incluyen la lectura, conferencias, escritura, trabajo en equipo, sueños diarios o trabajo en un equipo directivo (Cope, 2001). 
En otras palabras, los mecanismos para la creación de conocimiento personal consisten en una serie de pasos críticos donde se producen combinaciones de conocimiento tácito y explícito y este proceso se genera por medio del Aprendizaje Organizacional.

Los pasos críticos dentro del proceso de conversión de conocimiento son: exteriorización, interiorización, socialización y combinación.

En la fase de socialización es importante compartir experiencias; la externalización del conocimiento se genera mediante la creación de metáforas; en la combinación se comparte el conocimiento sistemático y en la internalización el conocimiento se aplica mediante el aprender haciendo.

Para la conversión del conocimiento de tácito en explícito y de explícito a tácito debe de existir interacción entre las diferentes formas de conocimiento, lo cual es fundamental para ir generando los ciclos de producción del (Figura 1).
Este proceso de creación del conocimiento se convierte en una espiral permanente de transformación ontológica interna de conocimiento, desarrollada mediante el seguimiento de las cuatro fases. En este modelo, el sentido de la dirección, es la esencia de cómo aplicar de la mejor forma posible un conocimiento existente para poder crear otro conocimiento nuevo o reciclado (Druker, 1993).

Tomando como base la propuesta efectuada por Nonaka y Takeuchi (1995) se muestra cómo el conocimiento individual, pasa a formar parte del conocimiento colectivo de la empresa, mediante el proceso de conversión del conocimiento (Figura 2).

En el campo de la salud pública, la producción de conocimiento en los servicios de salud, es permanente y se da en función a los problemas observados en el quehacer cotidiano, a la elaboración, aplicación y evaluación de soluciones para dichos problemas (Organización Panamericana de la Salud [OPS], 1992).

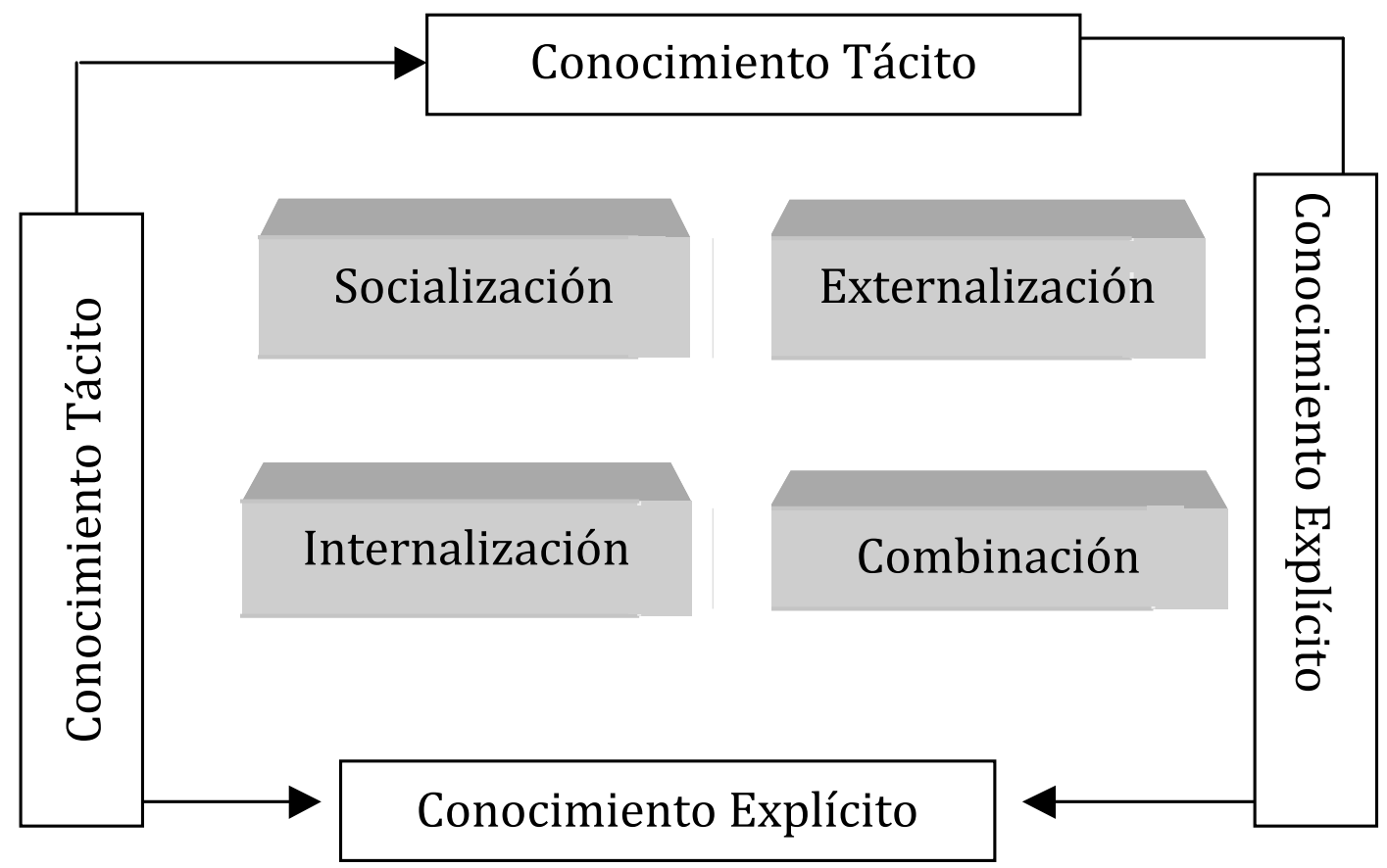

Figura 1. Mecanismos del conocimiento. Modelo planteado por Nonaka y Takeuchi (1995). 


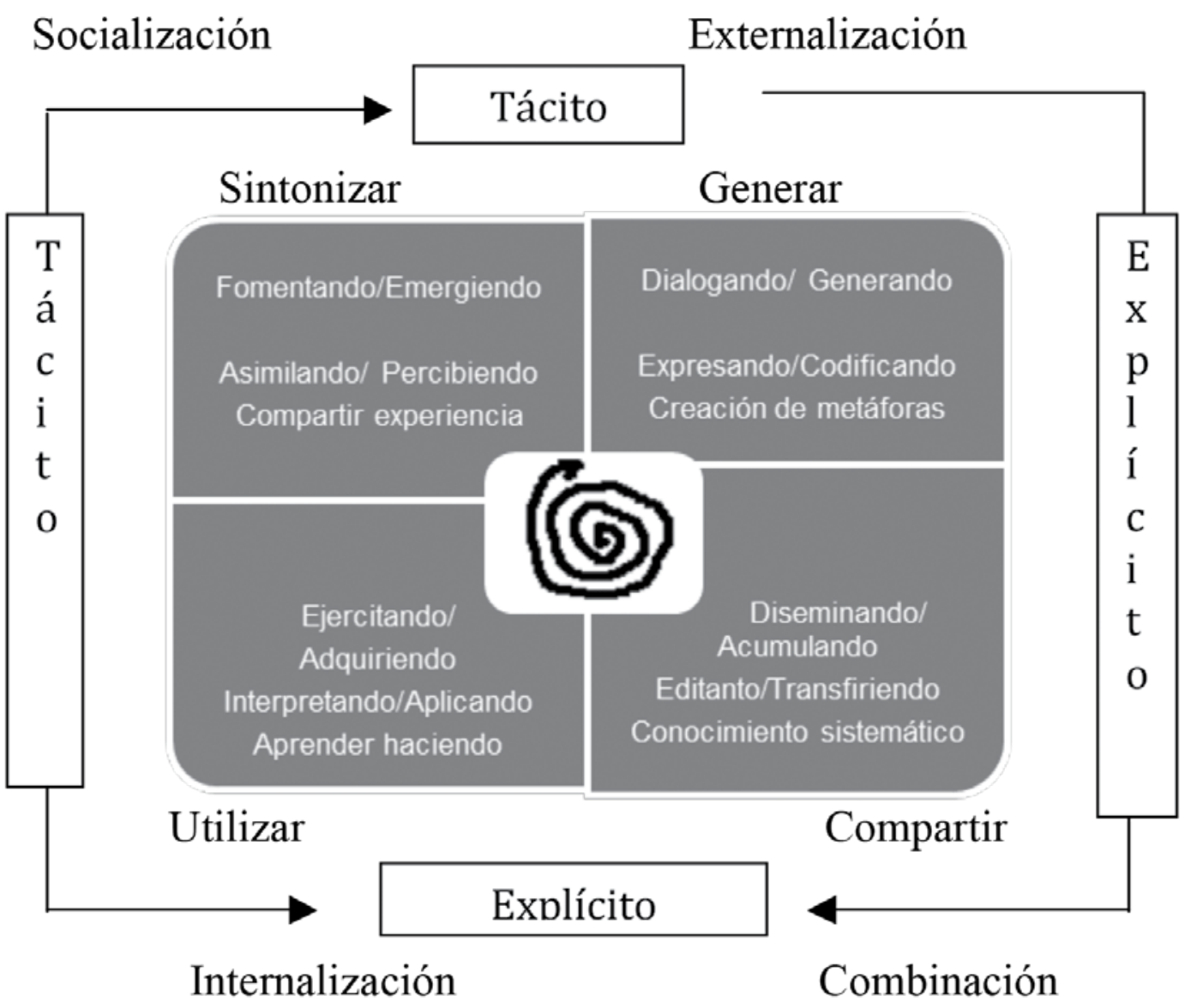

Figura 2. Interacción entre conocimiento tácito y explícito. Modelo de Ciclos de Producción del Conocimiento planteado por Nonaka (2007).

Por consiguiente, la gestión del conocimiento en un servicio de salud debe identificar y caracterizar el conocimiento existente ya sea tácito o explícito. Es por ello que la transferencia efectiva de conocimiento tácito, juega un importante rol para alcanzar las metas de desempeño. Es decir que "la transferencia del conocimiento tácito es facilitada por la relación interpersonal intensiva" (Máynez-Guaderrama \& Cavazos-Arroyo, 2011).

Ejemplo de lo anterior aplicado a la Salud Pública es el desarrollo de la OPS, que luego de la ratificación del Código Sanitario Panamericano en 1924, ha generado dinámicas de necesidad de nueva información y conocimiento para dar respuesta a problemas surgidos en el ámbito de la salud pública. Asimismo, derivado de la gestión de información y conocimiento se han originado nuevas formas de organización, planificación y dirección.
Ahora bien, el conocimiento puesto en práctica por los países de la región, ha hecho crecer la espiral del conocimiento hacia una retroalimentación y toda información local, estadísticas nacionales y el saber adquirido por experiencias nacionales y locales se ha convertido en saber e información universal y el poder del conocimiento generado ha sido reflejado en las resoluciones emanadas de las conferencias referentes a medidas sanitarias asumidas como conocimiento propio por parte de países miembros para mejorar la salud pública (Parra, 2005).

En esta experiencia la calidad de la comunicación, el manejo de la información y el conocimiento ha demostrado también cómo la comunidad de las Américas ha sabido aprovechar la comunicación humana como un activo. De igual forma, un establecimiento de salud su contexto de trabajo se convierte en un laboratorio de enseñanza-aprendizaje en donde los proveedores 
y usuarios, mediante la relación práctica, combinan información, contexto y experiencia, convirtiendo el conocimiento tácito en explícito y viceversa.

En el caso específico de la atención prenatal el proveedor transfiere el conocimiento tácito en la prestación del servicio y también el explícito al proveer información importante a la adolescente tanto para su autocuidado como en la identificación de signos y síntomas de riesgo en su embarazo.

De igual forma sucede con la adolescente primigesta, porque la importancia que dé a la información recibida va a depender del tipo de atención proporcionada, la empatía con el personal que le atendió y por supuesto, si la información proporcionada es acorde a su cultura, valores y creencias.

Por lo anterior, todo servicio de salud debe de contar con un análisis sociocultural de la población de su área de influencia para comprender las diferencias entre sus conocimientos, elementos terapéuticos y con base a ese conocimiento proporcionar los servicios, de tal forma, que la transferencia se desarrolle en forma efectiva.

De acuerdo con Cope (2001) el descubrimiento del conocimiento es el proceso mediante el cual se amplía la cantidad y calidad del contenido de conocimiento y la difusión es el proceso que se genera para compartirlo con el objetivo de ampliar o mejorar el valor y la calidad del mismo.

Una institución de salud, por ende, debe tener la capacidad de generar nuevos conocimientos y de difundirlos entre sus miembros para materializarlos en productos, servicios y sistemas que permitan solucionar los problemas de salud prevalentes.

El conocimiento como factor de producción debe buscar el desarrollo de tecnologías, metodologías y estrategias para que su medición, creación y difusión se convierta en prioridad para la organización como integrante de la sociedad del conocimiento. Así, el conocimiento se transforma en un elemento indispensable para su desarrollo económico y social.

La estructura del modelo de Nonaka y Takeuchi (1995) permite la generación de conocimiento colectivo, que se convierte en un valor agregado de la institución.

Si la salud pública se visualiza como algo social y no como problemas individuales, se hace indispensable cambiar el paradigma positivista que ha predominado a la fecha por una concepción de salud fundamentada como derecho humano y social, a ser garantizado por el Estado (Feo, Feo, \& Jiménez, 2012).
Es decir, los servicios de salud en el nivel local, deben convertirse en lugares estratégicos de construcción del orden social", para ello es necesario "desplazar capacidades de articulación y conducción del nivel central de la institución, como mecanismo de articulación con la sociedad civil (Romero, 2013).

Lo anterior requiere una descentralización o en su caso desconcentración de actividades del nivel central al local, lo que implica transferencia de conocimientos, competencias y servicios contextualizados a otros niveles regionales y locales. Un servicio es contextualizado cuando está enfocado en una visión integral de la cultura, valores y costumbres de cada comunidad; así como la comprensión e interpretación de determinantes sociales, dinámicas y procesos de su contexto (Romero, 2013).

En el 1986, en la ciudad de Ottawa, se realiza la primera Conferencia Internacional sobre la Promoción de la Salud. En esta actividad es reconocida la salud como producto integral determinado por un conjunto de factores biológicos, sociales y ambientales y se evidencia la necesidad de orientar la prestación de servicios a la satisfacción de las necesidades culturales de los individuos y comunidades; por lo que es fundamental la creación de vías de comunicación intersectoriales que abarque lo sanitario, social, político y económico. Lo anterior exige la generación de conocimiento para promover cambios en la educación y formación profesional y de esta forma producir el cambio de actitud y de organización de los servicios sanitarios (Organización Panamericana de la Salud /Organización Mundial de la Salud, 1986).

En la Carta de Ottawa de 1986, se reconoce la relevancia de las decisiones políticas de la salud, de la importancia de aunar esfuerzos y recursos sectoriales y de fortalecer la participación social en la búsqueda de un cambio en el estilo de vida y de la necesidad de proporcionar a los pueblos los medios necesarios para mejorar su salud y ejercer un mayor control sobre la misma y se afirma que la salud se crea en el contexto de la vida cotidiana (Romero, 2013).

Esta formulación del concepto de promoción de la salud que se presenta en la Carta de Ottawa significa construir políticas públicas saludables, crear un ambiente de apoyo, fortalecer la acción comunitaria, desarrollar aptitudes personales, y reorientar los servicios de salud (OPS, 2001).

Lo anterior indica que la actividad principal de la promoción de la salud debe de orientarse a la transferencia de resultados de investigación a los agentes 
sociales y en la gestión de procesos, por ello, los retos de la sociedad del conocimiento en la promoción de la salud debe ser: la integración de conocimiento en un entorno globalizado; la dinámica especialización-interdisciplinariedad; el filtrado y modulado de la información; el conocimiento de calidad; el aprendizaje continuo; la gestión de las redes del conocimiento. Por lo que el reto debe ser custodiar y comunicar conocimientos de calidad, diversos, integrados, dirigidos a receptores específicos en entornos de aprendizaje e innovación continuos con una estructura compleja gestionados por expertos (García, 2003).

Es decir que, en el campo de la salud pública, la demanda de servicios de salud en el mundo se ha incrementado en proporción directa con el crecimiento de la población y los problemas que la aquejan. En cambio, la oferta de servicios constituye una tarea compleja y ardua, dada la escasez de recursos financieros, las barreras tecnológicas y un factor cada vez más importante, la pérdida de competencias y desempeño del personal.

Incide también en este aspecto, la insuficiente capacidad gerencial de los gestores, además de la necesidad de que el servicio esté adaptado a las características culturales de cada país o nación. Es por ello que la gestión del conocimiento que se ha venido aplicando en el mundo empresarial, debe asumirse en el sector salud para mejorar la competencia y el desempeño de los individuos, los grupos y las instituciones.

Es decir que su finalidad debe ser optimizar el recurso humano para crear un capital humano o intelectual que unido a la gestión de calidad y otras herramientas gerenciales, permitan obtener servicios óptimos de salud, es decir, más calidad, accesibilidad y eficiencia. Consecuentemente, el estado de salud de la población debe elevarse pero el costo para las personas y las instituciones debe ser menor.

Mintzberg (1970), introdujo en la literatura administrativa una tipología organizacional con una visión innovadora para caracterizar y comprender las exigencias del trabajo en el sector salud.

Por ello, para lograr una gerencia de calidad existe la eminente necesidad de organizar los servicios de salud con eficiencia y efectividad (Navarro, 2004) y el óptimo que los sistemas de prestación deben alcanzar es la salud, mientras que los servicios obran, fundamentalmente, sobre la enfermedad.

Rojo (2003), refiere que los servicios de salud son espacios de realización de múltiples intereses, por lo que el desempeño del personal no puede regirse dentro del paradigma administrativo tradicional, porque es allí en donde confluyen instancias e intereses diversos de usuarios, profesionales, gestores, dirigentes políticos, gubernamentales, empresarios, suministradores de materias primas y la comunidad.

Esto significa que en un servicio de salud el conocimiento organizativo se debe crear, almacenar, difundir y reutilizar en el ámbito de toda la organización. Diferenciando el conocimiento como saber del de la habilidad, la cual se identifica como saber hacer (Valhondo, 2003).

Por ello, la gestión del conocimiento implica una mirada profunda hacia el interior de la organización de salud, de su personal calificado, de su desempeño, actitudes y es el análisis de la prestación del servicio de atención lo que permitirá identificar las brechas que al ser relacionadas con el parámetro que son las normas de atención vigentes evidenciará las necesidades de capacitación, normalización, de infraestructura, equipo, o de uso de tecnologías de información para el desempeño deseado del personal de salud que proporciona la atención.

La creación de la capacidad organizativa de un servicio de salud apoya la determinación, documentación, almacenamiento, distribución de recursos cognitivos; la capacidad de aprendizaje y de competencias que se generan y que son utilizadas por los individuos que la integran y comunidades en su área de influencia, en sus diferentes contextos profesionales (Organisation for Economic Co-operation and Development [OECD], 2003).

De acuerdo con Davenport y Prusak (1998, 2000), los factores que pueden determinar el éxito de un proceso de gestión del conocimiento deben estar orientados en poseer una cultura organizacional congruente a la producción y gestión del conocimiento, disponer de una infraestructura técnica e institucional y de canales existentes para la transferencia del conocimiento.

Sallis y Jones (2000), atribuye la gestión del conocimiento al contexto cultural, la confianza de los miembros de la organización, al liderazgo del personal directivo; así como a su coherencia con las estrategias establecidas.

Para Rivero (2002), el éxito dependerá de la existencia de un modelo común sobre gestión del conocimiento, la creación de una cultura común, del soporte tecnológico disponible y de no limitarse a realizar cambios cosméticos en la organización.

Alavi y Leidner $(1999,2001)$, lo dirigen a procurar que los datos sean utilizables, actuales y pertinentes, de asegurar la confidencialidad del cliente, de 
fomentar una cultura del conocimiento, establecer responsabilidades dentro de la organización con relación a la gestión del conocimiento y poseer infraestructura actualizada y segura.

Todo lo expuesto por los autores referidos puede resumirse en la interacción establecida con el usuario, la cultura organizacional existente y el liderazgo del personal directivo que es clave para la direccionalidad de las acciones.

Modelo propuesto para la transferencia del conocimiento en la atención prenatal a la adolescente primigesta (Figura 3)

Aplicando la teoría general de los sistemas, según Eiglier y Langeard (1990), el esquema para la producción de servicios, es similar al del sector de producción de bienes, en donde se necesitan: los recursos humanos que tiene relación directa con el usuario, el soporte físico que implica la infraestructura y equipo; la usuaria que es el elemento principal y el servicio como cuarto elemento en el sistema.

Esta herramienta metodológica permite enriquecer las acciones realizadas en un servicio de salud con el objetivo de satisfacer las necesidades de la usuaria en función a una atención prenatal integral, diferenciada y de calidad. El motivo de alcanzar esta satisfacción conlleva a que la adolescente use la información adquirida para incorporarla tanto en su auto cuidado como en el de su bebé.

La propuesta se ha realizado con base al triángulo del servicio que incluye aspectos clave del sistema de calidad y está en función de la satisfacción de la usuaria que surge de la interacción de la estrategia que responde al concepto del servicio, integración de equipo multidisciplinario funcional y de la estructura del sistema de prestación del servicio.

Para la operativización del modelo, es indispensable contar con servicios de salud integrales y diferenciados para la atención de la adolescente embarazada; así como definir las necesidades de la población a cubrir, de contar con la integración de un equipo multidisciplinario funcional mediante la estandarización y sistematización de la atención; para lo que se requiere de la realización de análisis de desempeño con el objetivo de identificar brechas y reducirlas por medio de la elaboración de un plan de acción que esté basado en parámetros establecidos en las normas y protocolos de atención existente.
Para este proceso es importante la aplicación de tecnologías que permitan la trasferencia del conocimiento y el desarrollo de habilidades del recurso humano que provee la atención prenatal. Esta transferencia de tecnología consiste en provocar el movimiento de información, o del conocimiento desde un proveedor hacia un receptor que en el caso de este ejemplo es la adolescente embarazada.

Mediante el uso de Tecnologías de Información y Comunicación se pueden generar cambios porque es el medio como las y los usuarios tienen acceso a la comunicación y la información, con intervenciones y acciones propuestas para la promoción y prevención de la salud.

Lo anterior, permite alcanzar mejores coberturas y la difusión de mensajes puede realizarse en forma interactiva, mediante redes sociales, chats, que actualmente son medios de comunicación en el grupo de adolescentes.

Asimismo, se puede hacer llegar la información en forma atractiva a las adolescentes y poder apoyarlas para que tengan conocimiento en cómo mantener su salud, identificar riesgos en forma oportuna y tomar decisiones informadas.

Con esas mismas herramientas tecnológicas también se pueden aclarar dudas o realizar cursos a distancia con contenido solicitados por los usuarios y de esta forma ir fomentando la salud de los diferentes grupos poblacionales en especial de los y las adolescentes y del cuidado del recién nacido.

Todo el proceso anterior debe estar en todas sus fases acompañado de un sistema de supervisión, monitoreo y evaluación institucional que acompañe el proceso implantado, permitiendo la toma de decisiones en forma oportuna en función del análisis de la información generada.

La gestión del conocimiento necesita de una adecuada gestión de la información, del uso apropiado de las tecnologías de información, de una correcta gestión de los recursos humanos y de enfoques novedosos de la práctica de la comunicación.

Para la conversión del conocimiento en la atención prenatal dirigida a adolescentes en un servicio de salud, se describen las cuatro formas de conversión del conocimiento tácito y explícito, mediante la adaptación del modelo de gestión del conocimiento de Nonaka y Takeuchi (1995) (Figura 4). En esta se explican las diferentes formas de conversión del conocimiento de tácito a tácito, tácito a explícito, explícito a explícito y de explícito a tácito en una institución que provee servicios 
de atención prenatal. Según el tipo de conocimiento, así será la metodología utilizada para su transferencia. Otro aspecto importante a considerar es la interacción posible entre las diferentes categorías definidas en las cuatro modalidades.

La conversión de tácito a tácito se realiza solo a través de la experiencia en forma de exposiciones orales, a través del diálogo o por medio de reflexión colectiva. Es producto de la interacción de las personas. Esta forma de conocimiento es novedosa para la base colectiva de la organización.
En el modo de combinación se crea conocimiento explícito que se puede categorizar y clasificar para producir una base de datos que posteriormente pueden generar conocimiento.

En la exteriorización el conocimiento se hace tangible por medio del uso de metáforas. Este conocimiento es difícil de comunicar, pero se integra a la cultura de la organización. Este modo es esencial en la creación del conocimiento.

La interiorización se produce cuando se analiza la experiencia de aplicación del nuevo conocimiento,

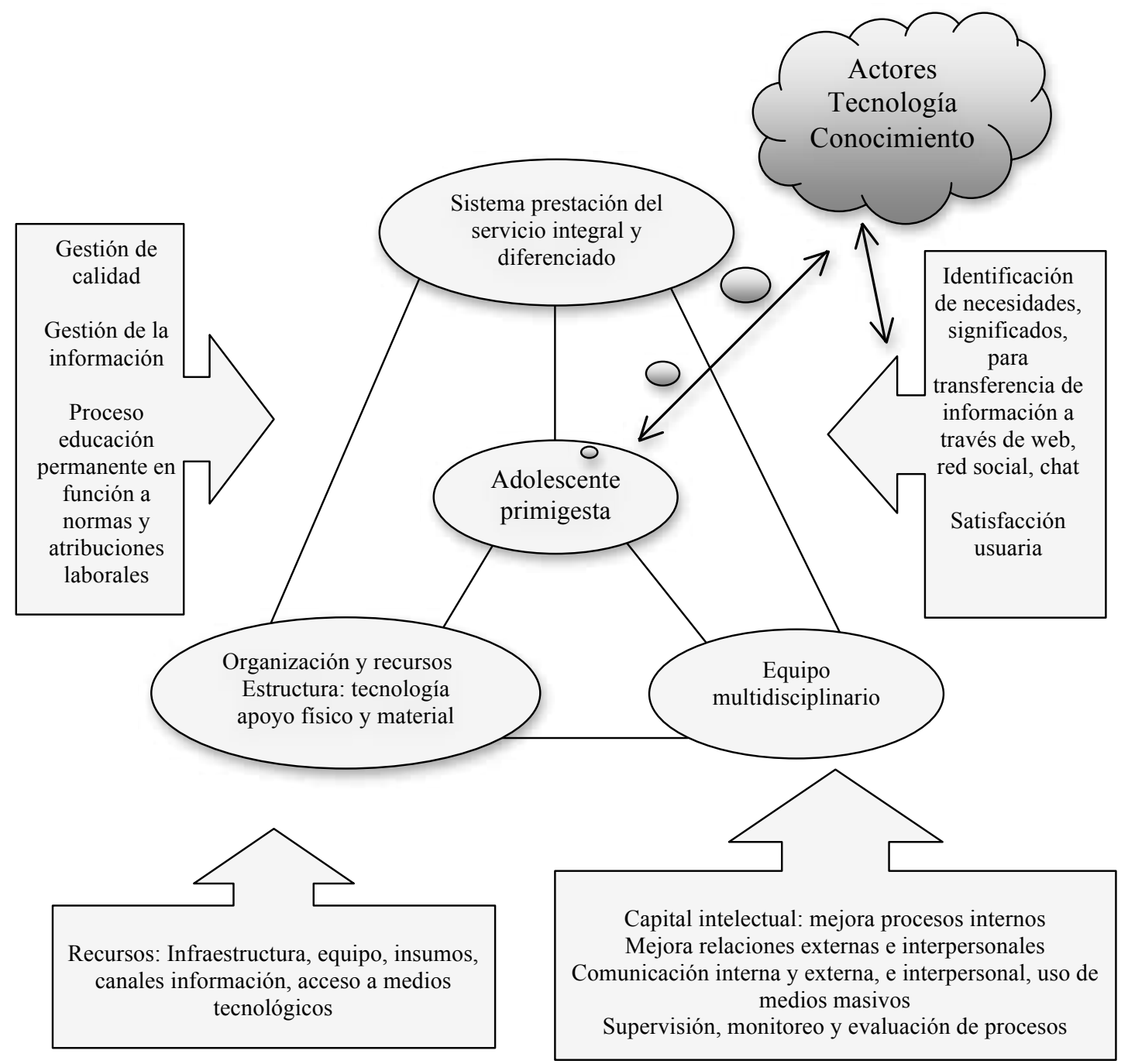

Figura 3: Propuesta de modelo para la transferencia del conocimiento en la atención prenatal de la adolescente primigesta. Adaptado de ISO (1991) y Albrecht (1992). 


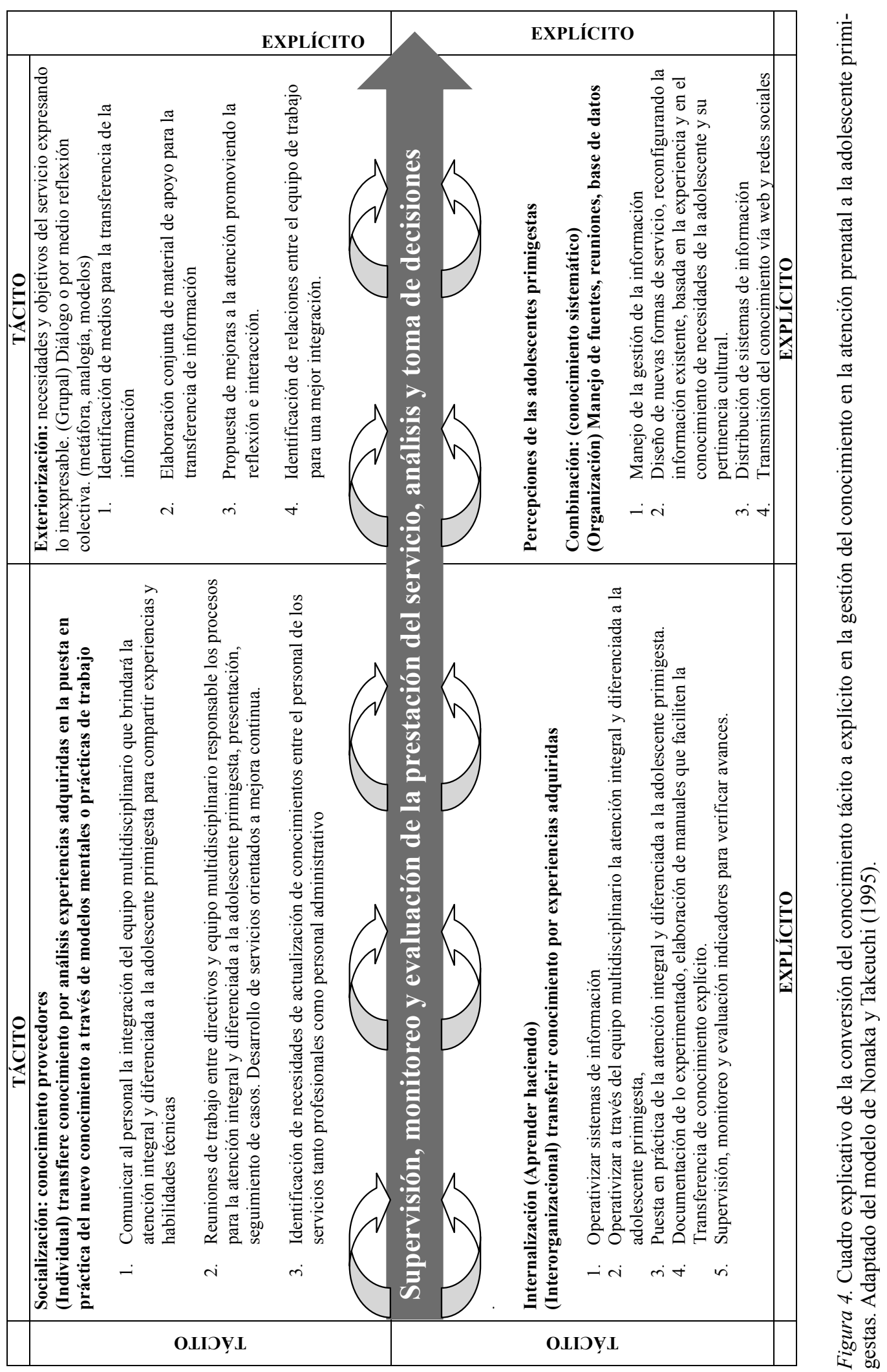


incorporándose el conocimiento generado al conocimiento tácito de los miembros de la organización.

\section{Conclusiones}

La gestión del conocimiento como proceso sistemático propicia espacios de interacción del recurso humano mediante el establecimiento de una red de relaciones que permite crear, organizar, transferir, almacenar y dar acceso al conocimiento generado para su utilización; permitiendo con ello una cultura de aprendizaje continuo entre los prestadores del servicio y con las usuarias, de tal forma que al utilizarlo se desarrollen competencias y capacidades para ser eficaces, productivos y portadores de una atención con calidez, contextualizada, de calidad.

Las instancias de Salud Pública deben propiciar mecanismos que activen la dimensión colectiva del conocimiento mediante la creación de una red institucional que permita la generación y transferencia del conocimiento en todos los niveles de atención de la institución, de tal manera, que influyan en la forma cómo se diseñan los puestos y procesos de trabajo orientados a la prestación de una atención prenatal integral diferenciada y con pertinencia cultural.

\section{Referencias}

Agencia Nacional de Infraestructura. (2012). Guía para la gestión del conocimiento. Código Gi-12. Bogotá: ANI. Recuperado de http://www.ani.gov. co/site/default/files/gi-12_guia_para_la_gestion del conocimiento v1 ani 0.pdf

Alavi, M., \& Leidner, D. (1999). Knowledge management systems: Issues, challenges and benefits. Communications of the, Association for Information Systems, 1(2), 1-35.

Alavi, M., \& Leidner, D. (2001). Review: Knowledge management and knowledge management systems: Conceptual foundations and research issues. Journal Management Information Systems, 25(1), 107-136.

Albrecht, K. (1992). La Revolución del Servicio. Santa fé de Bogotá: Legis Fondo Editorial Serie Empresarial.

Archanco, R. (2013). Breve guía no convencional para la gestión del conocimiento empresarial. Recuperado de http://papelesdeinteligencia. com $/ ? \mathrm{~s}=\mathrm{http} \% 3 \mathrm{~A} \% 2 \mathrm{~F} \% 2 \mathrm{Fpapelesdeinteligencia.}$ com $\% 2 \mathrm{~F}+$ gestion-del-conocimientoempresarial $\% 2 \mathrm{~F}$

Argote, L., \& Ingram, P. (2000). Knowledge transfer: A basis for competitive advantage in firms. Organizational Behavior and Human Decision Processes, 82, 150-169. doi:10.1006/ obhd.2000.2893

Boisier, S. (2002). Sociedad del conocimiento, conocimiento social y gestión territorial. Revista del Cesla, (4), 60-94.

Bürkli, A. (2011). Taller de Gestión del Conocimiento. Recuperado de https://ch4rm3d.files.wordpress. com/2011/09/gesti_n_del_conocimiento1.ppt

Cope, M. (2001). El conocimiento personal: Un valor seguro. Gestione su conocimiento y sáquele partido. Madrid: Prentice Hall.

Davenport, T., \& Prusak, L. (1998). Conhecimento Empresarial: Como asorganizações gerenciamo seu capital intelectual. Rio de Janeiro: Campues.

Davenport, T., \& Prusak, L. (2000). Working knowledge: how organizations manage what they know. Boston: Harvard Business School Press.

Drucker, P. (1993). Post-capitalist society. Oxford: Butterworth Heinemann.

Eiglier, P., \& Langeard, E. (1990). Servucción: El marketing de los servicios. Madrid: McGraw Hill.

Feo, O., Feo, C., \& Jiménez, P. (2012). Pensamiento contrahegemónico en salud. Maracay, Venezuela: Universidad de Carabobo.

Fernández, J. (2000). Los campos de acción de la gestión del conocimiento. Capital Humano, 124, 64-65.

García, F. (2003). La gestión del conocimiento: Aplicación a la promoción de la salud. Scire, 9(1), 151-170.

Gibbons, M., Limoges, C., Nowothy, H., Sschwartzman, S., Scott, P., \& Trow, M. (1997). La nueva producción del conocimiento. La dinámica de la ciencia y la investigación en las sociedades contemporáneas. Barcelona: Pomares-Corregidor.

Gil-Montelongo, M. D., López-Orozco, G., MolinaGarcía, C., \& Bolio-Yris, C. A. (2011). La gestión de la información como base de una iniciativa de gestión del conocimiento. Ingeniería Industrial, 32(3), 231-237. 
Grant, R. (1996). Prospering in dynamically competitive environments: Organizational capability as knowledge integration. Organization Science, 7(4), 375-388.

Koontz, H., \& Weihrich, H. (1998). Administración una perspectiva global. México, D. F: McGraw-Hill.

Lundvall, B.-A. (1999). La base del conocimiento y su producción. Ekonomiaz:Revista Vasca de Economía, (45), 14-37.

Martínez, M., (2011). Desarrollo de un modelo de gestión del conocimiento en la cadena de suministro de la industria agroalimentaria (Tesis de doctorado). Recuperada de http://oa.upm. es/6199/1/MOISES_ENRIQUE_MARTINEZ_ SOTO.pdf

Máynez-Guaderrama, A., \& Cavazos-Arroyo, J. (2011). Conocimiento tácito: Su transferencia dentro de la organización, como fuente de ventaja competitiva sostenible. Administración y Organizaciones, 14(26), 9-26.

Mintzberg, H. (1970). Structured observation as a method to study managerial work. Journal of Management Studies, 7(1), 87-104. doi: 10.1111/ j.1467-6486.1970.tb00484.x

Navarro, R. (2004). Factores socio-laborales que caracterizan la fluctuación laboral real. (Tesis de maestría). Escuela Nacional de Salud Pública, Cuba. Recuperado de http://www.sld.cu/galerias/ pdf/sitios/revsalud/tesisray.pdf

Nonaka, 1., \& Takeuchi H. (1995). The knowledgecreating company. Oxford: Oxford University Press.

Nonaka, I. (2007). The Knowledge-Creating Company. Harvard Business Review, 85(7/8), 162-171.

Organisation for Economic Co-operation and Development. (2003). Measuring knowledge management in the business sector: First steps. Paris: Autor.

Ordoñez, P. (12 -14 de septiembre 1999). Gestión del conocimiento y medición del capital intelectual. Presentado en el IX Congreso Nacional de la Asociación Científica de Economía y Dirección de la Empresa, Burgos.

Organización Panamericana de la Salud /Organización Mundial de la Salud. (1986). Carta de Ottawa para la promoción de la salud, primera conferencia internacional sobre promoción de la salud. Otawa, Canadá: Autor.

Organización Panamericana de la Salud. (1992). Desarrollo y fortalecimiento de los sistemas locales de salud. La administración estratégica. Washington, D.C: Autor.

Organización Panamericana de la Salud. (2001). Informe anual del director. Promoción de la salud en las Américas (Documento oficial No. 302). Washington, D.C: Autor.

Parra, R. (2005). Gestión de información y conocimiento en salud pública y la usabilidad en la Web en comunicación para el desarrollo. Presentado en el Congreso Internacional Online TIC vida. Bogotá, Colombia.

Red Colombiana de Investigación en Política y Sistemas de Salud. (2009). La gestión del conocimiento para la salud pública. Plan Estratégico de Antioquia. Antioquia, Colombia: Autor. Recuperado de http://www.es.slideshare. net/radarik/la-gestion-del-conocimiento-para-lasalud-publica

Rivero, S. (2002). Claves y pautas para comprender e implantar la gestión del conocimiento: Un modelo de referencia. Las Arenas: Socintec.

Rodríguez, D. (2006). Modelos para la creación y gestión del conocimiento: Una aproximación teórica. Educar, (37), 25-39.

Rojo, N. (2003). Administración de recursos humanos. Generalidades en salud. En O. Carnota, Biblioteca Virtual para formación postgraduada de directivos del sector salud [CD-ROM]. La Habana, Cuba: Escuela Nacional de Salud Pública.

Roldán, J. (2000). Sistemas de información ejecutivos (EIS): Un estudio descriptivo sobre la situación en España. Presentado en el I Encuentro Iberoamericano de Finanzas y Sistemas de Información. Universidad de Cádiz, Jerez de la Frontera, España.

Romero, C. (2013). La construcción social de la salud y los gobiernos locales. Costa Rica: Universidad de Costa Rica. Recuperado de www.binasss.sa.cr /revistas/ts/63/art3.htm/04/2014

Sallis, E., \& Jones, G. (2002). Knowledge management in education: Enhancing learning and education. London: Kogan Page. 
Sakaiya, T. (1991). The knowledge-value revolution or a history of the future. Tokio, Japan: Kodansha.

Senge, P. (2011). La quinta disciplina. El arte y la práctica de la organización abierta al aprendizaje. Buenos Aires, Argentina: Granica.

Serradell, E., \& Juan, A. A. (2003). La gestión del conocimiento en la nueva economía. Universitat Oberta de Catalunya. Recuperado de https://www. uoc.edu/dt/20133/20133.pdf

Sveiby, K. (2001). A knowledge-based theory of the firm to guide strategy formulation. Journal of Intellectual Capital, 2(4), 344-358. doi: 10.1108/14691930110409651
Urra, P. (2008). Reflexiones en torno a la gestión del conocimiento en salud. Presentación Congreso Internacional INFO 2008. Habana, Cuba. Recuperado de http://www.sld.cu/galerias/pdf/ sitios/vigilancia/urra.pdf

Valhondo, D. (2010). Gestión del conocimiento: Del mito a la realidad. Madrid: Díaz de Santos.

Woodman, L. (1985). Information management in large organisations. Information management from strategies to action. London: B. Cronin. 